\title{
Diagnóstico para la propuesta curricular de la Carrera de Gestión del Conocimiento en la modalidad a distancia y la aplicación del método
}

\author{
Javier Francisco GARCÍA OROZCO \\ Universidad de Guadalajara \\ México
}

Recibido: 16/02/2011

Aceptado: 9/03/2011

\section{RESUMEN}

En el presente articulo se presentan los resultados del diagnostico para la propuesta de diseño curricular de la carrera de Gestión del Conocimiento (GC) como programa a distancia, haciendo énfasis en la construcción colectiva del mismo a través de la metodología de grupos focales.

Palabras clave: Diseño Curricular. Gestión del Conocimiento (GC), Educación a Distancia, Grupos Focales.

\section{Diagnosis for the proposed curriculum of the School of Knowledge Management in the distance mode and the method}

\begin{abstract}
In this paper to present the results of the diagnostic for the proposed curriculum of the career of Knowledge Management (KM) as a distance learning program, focused to the collective construction through metodology of the focus groups.

Key words: curriculum design, Knowledge Management (KM), Distance Education, Focus Groups.
\end{abstract}

\section{INTRODUCCIÓN}

La información y el conocimiento son parte de un mismo proceso complejo vinculado por conceptos como aprendizaje, inteligencia y tecnologías. Los flujos, la información y la generación de nuevos conocimientos dependen de modelos de comportamientos informacionales y cognitivos de las personas.

Buscar, almacenar, procesar, analizar, representar, compartir y distribuir información y conocimiento debe ser una labor relacionada con la motivación y el estimulo de los usuarios y por ende con la creación de competencias y habilidades que se han de formar de manera sistemática.

La información y el conocimiento se trasmiten e intercambian a menos costo para una cantidad mayor de usuarios a la vez, de forma estructurada y formalizada y a través del uso de las tecnologías de información. Los soportes de la información pueden ser muy diversos, el soporte fundamental del conocimiento son las personas. 
En los países de habla hispana esto aun poco difundida la labor profesional de los gestores de conocimiento, aunque en realidad se realiza sistemáticamente, ya que se confunde frecuentemente por parte de los directivos al ingeniero en sistemas informáticos con el gestores y creadores de sistemas de información y conocimiento, y peor aun, no se identifica el objeto de trabajo de cada uno de ellos, considerando que solo los sistemas son la solución de problemas y no la gestión inteligente de los mismos.

El presente trabajo presenta los resultados del diagnostico realizado para el la propuesta curricular de la carrera de Gestión del Conocimiento del Sistema de Universidad Virtual de la Universidad de Guadalajara a partir de grupos focales,

\section{La carrera de Gestión del Conocimiento}

En el documento del la OCDE "Panorama de la Educación 2010" se plantea:

Los sistemas educativos, que con frecuencia han tendido a operar en función de la oferta, habrán de desarrollar mecanismos eficaces para entender unas demandas sociales y económicas de competencias en constante mudanza y responder a ellas. Las políticas eficaces en este campo requieren un sólido entendimiento no solo del desarrollo de las competencias, sino también del grado de eficacia de las economías en el uso de su reserva de talento y del modo en que una mejora de las competencias se traduce en mejores trabajos, una productividad más alta y, por último, mejores resultados sociales y económicos.....

y continúa:

Los ciudadanos y los empleadores de hoy esperan que los sistemas educativos:

- respondan a demandas cambiantes, garantizando que la educación y los proveedores de formación se adaptan eficientemente a ellas;

- aporten calidad y eficiencia en la provisión del aprendizaje, de manera que se adquieran las competencias adecuadas en el momento adecuado, en el lugar adecuado y de la manera más eficaz;

- proporcionen la flexibilidad necesaria para que las personas puedan estudiar y formarse en lo que deseen, cuando quieran hacerlo y de la manera en que quieran hacerlo;

- reduzcan obstáculos para acceder a la educación, como las rigideces institucionales, las tasas por anticipado y las restricciones de edad, y garanticen suficiente variedad de vías de acceso y nuevo acceso; y

- desarrollen perspectivas sostenibles y eficientes a la financiación del aprendizaje con una base racional para quién debe pagar.....

En este nuevo contexto la formación en áreas relacionadas con la Gestión del Conocimiento es imprescindible.

La Gestión del Conocimiento es un área emergente de las ciencias que como muchas tiene un carácter multidisciplinar que toca elementos relacionados con 
ciencias de la información, la administración de las organizaciones, la gestión cultural, la comunicación y la aplicación de tecnologías de información.

Sin embargo, los empresarios del sector privado y directivos públicos aun no visualizan el lugar de un experto en esta área como necesario para su staff, por lo que la demanda de estos profesionales es aun incipiente y requiere de un impulso por parte de la instituciones académicas las cuales deben formar especialistas que sepan manejar la información y el conocimiento como valor real de las organizaciones alcanzando ventajas competitivas para las mismas.

El estudio de la Gestión del Conocimiento es abordado desde diversas perspectivas, y se han propuesto muy diversos modelos vinculado a la vertiente económica de la GC, una de las cuales, tiene la intención de identificar las problemáticas relacionadas con su implementación en los diferentes sectores productivos. (Davenport, 2000; Nonaka \& Takeuchi, 1995; Sveiby, 2000; Polanyi, 1998; Duncker 2007)

A este respecto, el diagnostico se basa en algunas premisas, las cuales son imprescindibles de tener en cuenta para el desarrollo del trabajo. Estas son las siguientes:

1. Es necesario generar una transición hacia un nuevo sistema económico, basado en la información y el conocimiento, como insumos de valor para los procesos de producción y servicios.

2. No existe un acceso estructurado y sistémico a la información y construcción del conocimiento, en nuestro contexto y es evidente el desconocimiento del valor de estos insumos y su manejo dentro de las organizaciones.

3. La productividad y competitividad de las organizaciones dependen del acceso oportuno a la información y difusión del conocimiento para la innovación continua en productos y servicios.

4. Existe un atraso en la generación y aplicación de conocimientos propios por parte de las organizaciones, no se generan patentes ni transferencias tecnológicas de avanzada.

5. No existe capacidad para desarrollar tecnologías convergentes y de avanzadas dentro de las organizaciones.

6. La Gestión de la Información y el Conocimiento debe permitir que la organización reaccionen más rápida y eficazmente a los cambios del entorno en que opera.

7. La Gestión de la Información y el Conocimiento debe actuar como motor de cambio dentro de la organización, y anticipar los cambios en la cultura de la misma.

\section{Diseño curricular por competencias}

El diseño curricular puede concebirse como un espacio del curriculum que propone la metodología, las acciones y el resultado del diagnóstico, modelación, y organización de los proyectos curriculares. 
El mismo parte de un modelo educativo determinado y específico que al operar, pretende solucionar problemas y satisfacer necesidades y a través de su posterior evaluación, posibilita el mejoramiento del proceso de enseñanza-aprendizaje.

El diseño curricular como metodología explica cómo elaborar la concepción curricular; como acción constituye un proceso; y como resultado quedan plasmados en guías y documentos que presentan dicha concepción y las formas de ponerla en práctica y evaluarla.

En el Sistema de Universidad Virtual de la Universidad de Guadalajara se toma como metodología base para el diseño curricular la propuesta de Chan Núñez (2003), de diseño curricular por competencias la cual incluye una serie de instrumentos que permiten estructurar adecuadamente la captura y análisis de información.

La propuesta, ya probada en numerosas ocasiones en la práctica, incluye las siguientes fases fundamentales:

1. Búsqueda bibliográfica sobre problemáticas de la profesión que se propone

2. Identificación de los problemas y perfil deseable

3. Identificación de competencias

4. Definición de las funciones integradas en las competencias y ámbitos de desempeño

5. Desagregación de la competencia en conocimientos, atributos, habilidades y valores

6. Mapa curricular considerando diferentes escenarios de trayectorias escolares

Tobon, (2006) propone como concepto de competencias el siguiente:

Procesos complejos de desempeño con idoneidad en un determinado contexto, con
responsabilidad.

El autor, uno de los mas importante en este tema en la actualidad, refiere la complejidad de este concepto el cual ha sido objeto de diversas criticas por una parte de diversos círculos académicos, tratando, desde un enfoque reduccionista, de desvirtuarlo, en virtud de los temores naturales al cambio y al desconocimiento sobre el tema.

El enfoque por competencias de diseño curricular, en el caso del Sistema de Universidad Virtual, es doblemente pertinente, por una parte satisface las demandas de los ciudadanos y empleadores a los sistemas educativos, citadas en paginas anteriores (OCDE, 2010) y por otra permite enfocarnos en el diseño instruccional de las materias a la generación de productos que evidencian el aprendizaje de los estudiantes, sin recurrir a métodos tradicionales de evaluaciones áulicas utilizados hasta el momento

Si se conjugan adecuadamente el enfoque por competencias en el hacer y el ser en el curriculum, este estará dando respuesta a la reclamación de una formación más adecuada de nuestros profesionales en la era de la información y el conocimiento y será el primer paso a vincular el aprendizaje a contexto reales de solución de problemas 


\section{METODOLOGÍA}

\section{Estudio de referencias de programas educativos}

Con la finalidad de identificar los principales programas educativos existentes relacionados con la gestión del conocimiento (GC), se llevó a cabo un estudio de referencias de estos programas en la Web

El estudio se inicia con una búsqueda exhaustiva de programas relacionados con la GC en México, España, Chile, Colombia, Argentina y Cuba, a través de Internet.

Se diseñó un formato para la captura de información d y se realizó la búsqueda considerando el nombre y el tipo de programa, la institución que lo ofrece, el país y el sitio Web.

En esta primera búsqueda se recuperaron 42 programas que conformaron una base de datos. Posteriormente, se realizó un filtraje de esta base de datos, considerando aquellos programas en los que la información estuviera vigente, actualizada y completa para efectos de este estudio, resultando que se consideraron pertinentes 19 programas de México, España, Chile, Colombia, Argentina y Cuba, incluyéndose además un programa de Uruguay.

\section{Grupos Focales (GF)}

La metodología utilizada para el estudio diagnostico, fue la relacionada con la participación de expertos en Grupos Focales a partir del Modelo Socio Céntrico.

Aigneren (2002) citando a Korman define un grupo focal como: "una reunión de un grupo de individuos seleccionados por los investigadores para discutir y elaborar, desde la experiencia personal, una temática o hecho social que es objeto de investigación".

El mismo autor señala que los GF se caracterizan por la interacción, discusión y elaboración de unos acuerdos dentro del grupo acerca de unas temáticas que son propuestas por el investigador Chan Núñez (2004) plantea que el diseño curricular es una tarea colectiva que redundara en una visión mas integral del producto a desarrollar, es por ello que asumimos como valido la conformación de grupos focales para es desarrollo del diagnostico, la estructuración de competencias y la organización de la malla curricular.

Para la conformación de los grupos focales, fue necesario clasificar el universo de participantes líderes de opinión, en los sectores empresarial, investigación, académico, agrícola, gobierno y salud, quienes participaron en una sesión colaborativa de trabajo.

El objetivo fundamental del grupo focal es alcanzar o lograr el descubrimiento de una estructura de sentido compartida, si es posible consensualmente, o, en todo caso, bien fundamentada por los aportes de los miembros del grupo.

Se conformaron 3 mesas de trabajo, cada una de ellas integradas por representantes de diferentes sectores, un moderador y dos relatores. Estos tres últimos integrantes se encargaron de la coordinación, participación y recolección de datos 
obtenidos en los grupos exclusivamente. Se grabaron y filmaron todas las sesiones de trabajo completas.

El moderador no participó en la producción de la ideas, ni, mucho menos, evalúo, aprobó o desaprobó, el contenido de lo que fue apareciendo; sólo guió la reunión dando la palabra, llevando la conversación hacia la temática en cuestión, sin permitir digresiones serias, pidió que concretaran o integraran sus ideas sin dispersión.

El moderador manejo la aparición de líderes y su integración o ajuste en sus planteamientos. Se tuvo en cuenta un tiempo estricto de dos horas para el debate y participación de los miembros del grupo y se estableció un guión para orientar la discusión.

La información recogida producto de los debates sobre las necesidades y la problemática en cuestión, de los participantes de los grupos focales, fue recopilada en documentos escritos, audio y videos, que posteriormente fueron material de referencia para elaborar una matriz FODA e identificar la problemática y necesidades expuestas.

Se realizó una síntesis por cada una de las tres mesas, con su respectiva matriz y lista de necesidades. Posteriormente estas matrices y listas fueron compiladas en un solo documento para obtener un informe completo de cada uno de los grupos participantes.

Este documento, fue revisado por un grupo de 3 expertos en el área de gestión del conocimiento, quien nominalizó los ejes y las variables a partir de la lista de necesidades

Posteriormente, se utilizó el método del valor esperado, usando la técnica NGT (Nominal Group Technique), para identificar resultados cuantitativos de cada una de las variables por eje, aplicando pesos y escenarios. Este método arrojó como resultado, tres escenarios diferentes, los cuales sirvieron de modelo para identificar valores medios de cada uno de los ejes y minimizar la subjetividad de los analistas.

\section{$\underline{\text { Análisis de los resultados }}$}

A continuación se presentan el análisis de los resultados obtenidos dividido en el análisis de los programas existentes consultados y lo relacionados a las necesidades y demandas de proveedores, o sea participantes de los grupos focales

\section{Programas Educativos en Iberoamérica}

La información recuperada se completó con el estudio de los aspectos relacionados con los objetivos de los programas, contenidos, planes de estudios y modalidad de los programas, entre otros datos relevantes.

De este estudio se obtuvieron los siguientes resultados: 


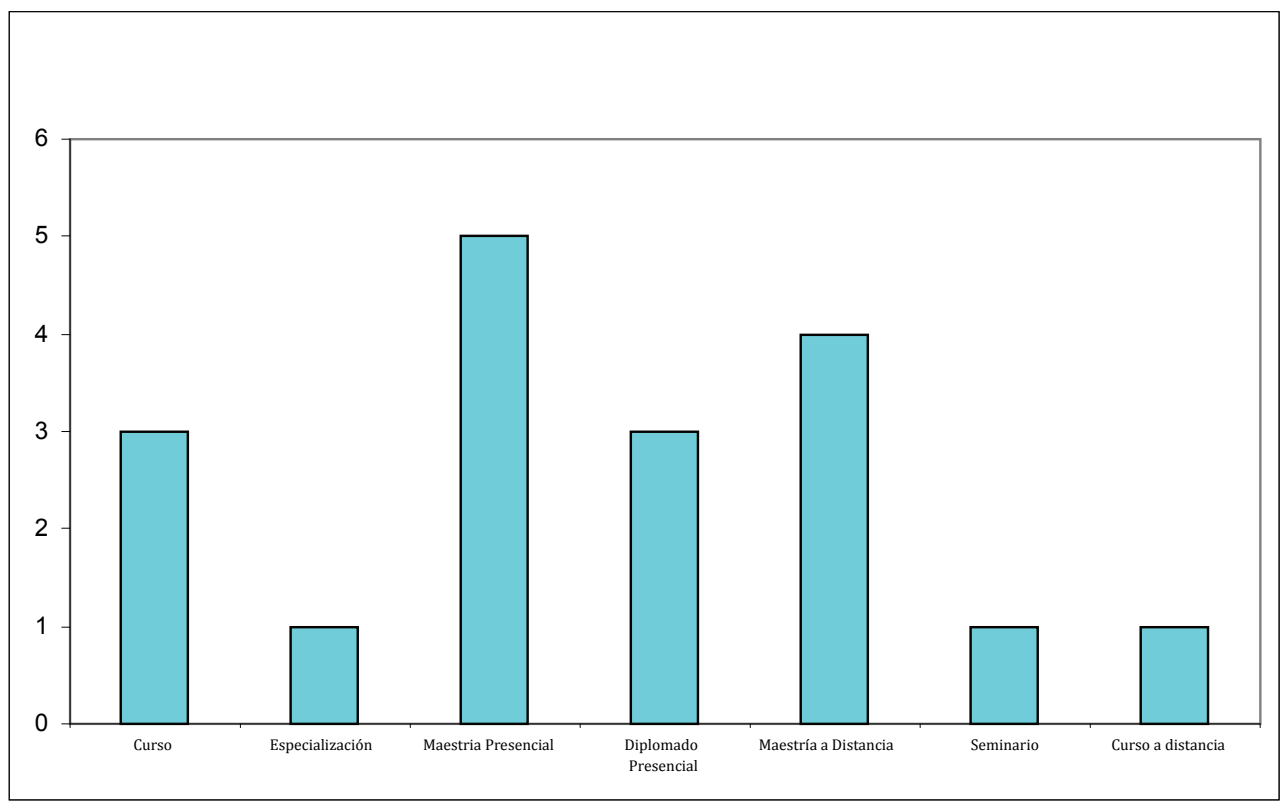

Fig. 1 Oferta educativa a distancia en GC en America Latina y el Caribe

Como se observa en la figura 1 ninguno de los programas analizados, se desarrolla a nivel licenciatura, lo que representa un espacio de oportunidad si se quisiera incursionar en este tipo de programa. En nuestra opinión el área de GC debe desarrollarse a nivel postgrado debido a que una formación base se encuentra en las especialidades relacionadas con la misma a saber: Información, Administración, Gestión Cultural y Tecnologías de Información. Esta opinión personal ha sido compartida por un numero grupo del Sistema de Universidad Virtual ${ }^{1}$

Es posible que la ausencia de expertos y académicos con experiencia sobre la aplicación práctica de sistemas de gestión del conocimiento en las organizaciones, sea la razón por la cual la formación de especialistas cercanos a la presente propuesta este ausente a nivel licenciatura. Hasta ahora la formación se ha vinculado a la administración de unidades de información en las carreras de bibliotecología o de ciencias de la información, o a especialistas de carreras con un perfil estrictamente tecnológico que se enfocan a la programación de sistemas informáticos, quedando un vacío en esta área emergente del conocimiento

\footnotetext{
${ }^{1}$ Para discutir la metodología y el planteamiento de la carrera se celebró una reunión de análisis con coordinadores de carreras e investigadores del Sistema de Universidad Virtual los cuales por consenso expresaron su criterio de que un programa de GC debería ser de postgrado
} 


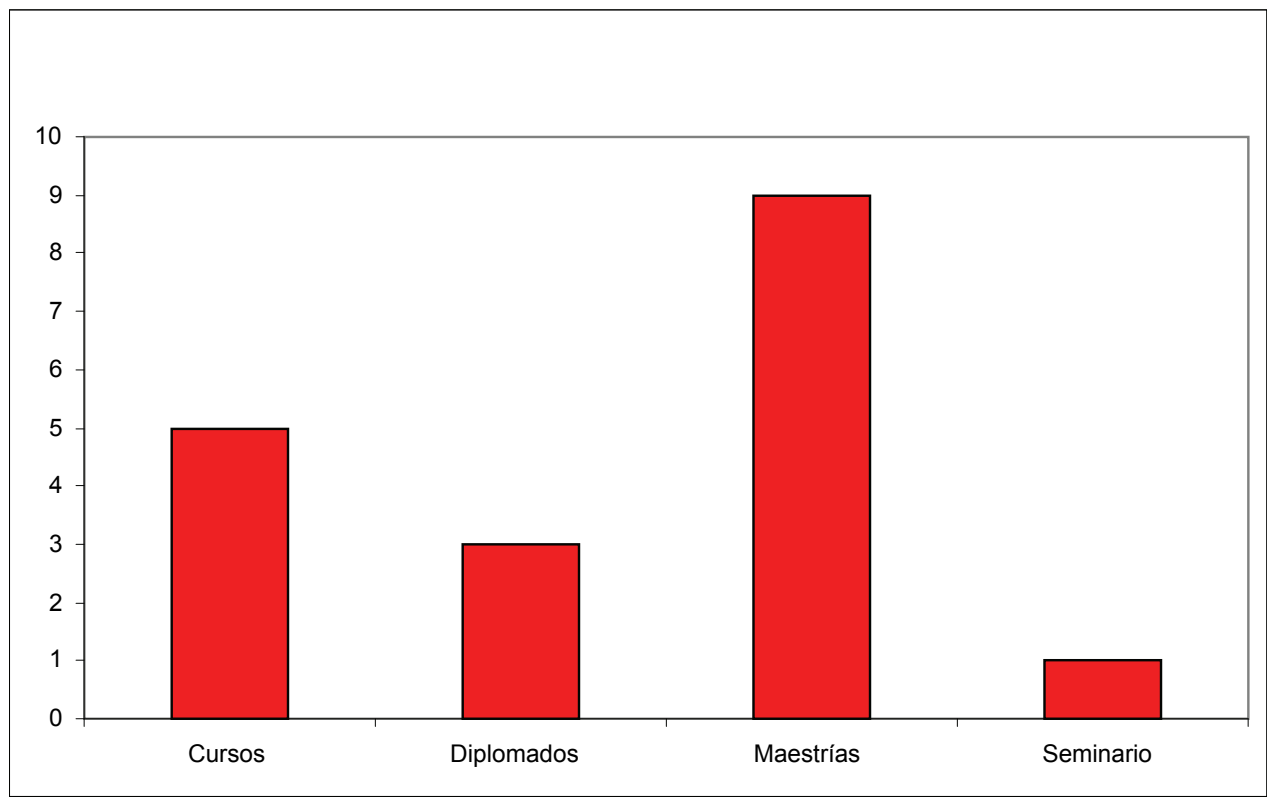

Fig. 2. Tipología de la oferta educativa

En la figura 2 se evidencia que el nivel de maestría es el que mayormente se ofrece, seguido por cursos de especialización, diplomados y por último seminarios.

La explicación a este hecho puede ser la misma que se señala en el párrafo anterior, o sea la falta de expertos y experiencia profesional. 


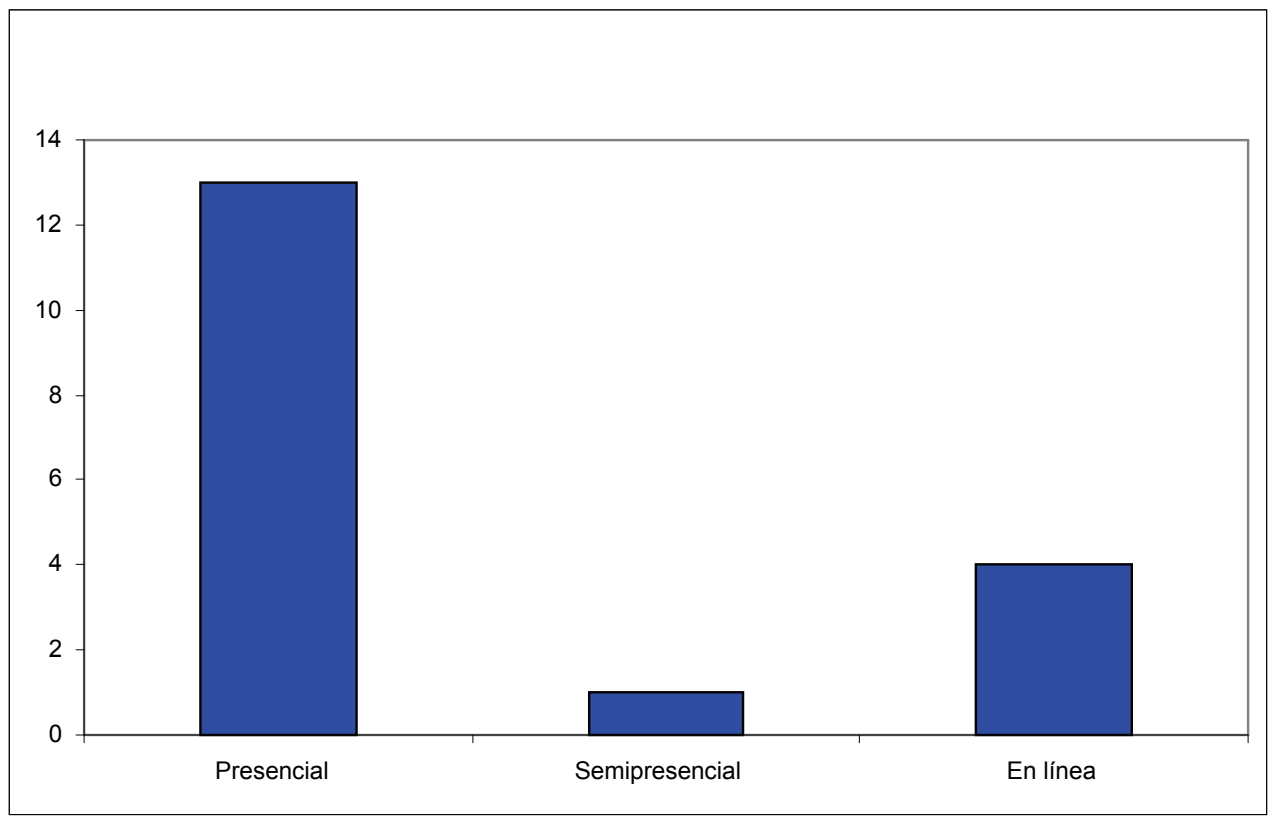

Fig. 3 Distribución por modalidad educativa

Como se observa en la figura 3 los programas se desarrollan primordialmente en modalidad presencial, seguido de los programas en línea, y por último los semipresenciales o híbridos, evidenciando un campo de oportunidad para el desarrollo de programas a distancia.

De estos resultados, se observan que las instituciones educativas, son cada vez más conscientes del valor que aportan los activos intangibles a las organizaciones (la mayoría de estos activos, están basados en la información, el conocimiento y el aprendizaje) y cada vez mas recurren a la formación de postgrado en esta especialidad o a cursos de especialización

Los programas educativos detectados y analizados responden a:

- necesidad de preparar directivos de alta calificación para el manejo y aprovechamiento de los recursos tecnológicos e informativos.

- desarrollado de vínculos profundos a los nuevos paradigmas de las tecnologías de la información y el conocimiento.

- los ejecutivos y profesionales que tienen a su cargo el manejo recursos humanos, financieros, materiales o técnicos y que están preocupados por el manejo de la información dentro de sus organizaciones y por la interacción entre los elementos humanos de la misma, siendo estos poseedores de conocimientos relevantes.

Estos programas educativos tienen el propósito de: 
1. Contribuir a la formación de competencias profesionales de directivos, ejecutivos y profesionales relacionadas con conceptos e instrumentos metodológicos de gestión del conocimiento y su aplicación en las organizaciones.

2. Mostrar el proceso de transformación que existe en las organizaciones actuales, visualizando las tendencias futuras y proporcionando bases para enfrentar los retos, considerando el cambio cultural y tecnológico permanente.

Los contenidos están enfocados a:

- Las teorías, procesos y fases de la gestión del conocimiento

- Innovación y competitividad

- Análisis de datos y de información

- Desarrollo de capital humano

- Comportamiento y aprendizaje organizacional

- Análisis y diseño de sistemas de información

- Técnicas de apoyo a la gestión de la información y el conocimiento

- Capital intelectual

- Usabilidad de la información

\section{Análisis de la problemática y las necesidades detectadas}

El primer eje de información que se detectó fue el de Gestión de información en el que se destacan los siguientes temas relevantes sobre necesidades y problemática:

- Gestión y análisis de la información

- Diagnóstico y almacenamiento

- Sistematización y registro

- Uso de la información

- Calidad de la información

- Accesibilidad y responsabilidad

- Clasificación y seguridad

- Distribución y comunicación.

Como se observa en la siguiente gráfica, los participantes centraron su atención en la gestión de la información en comparación con otros ejes, siendo este eje prioritario en las organizaciones para las que laboran. 


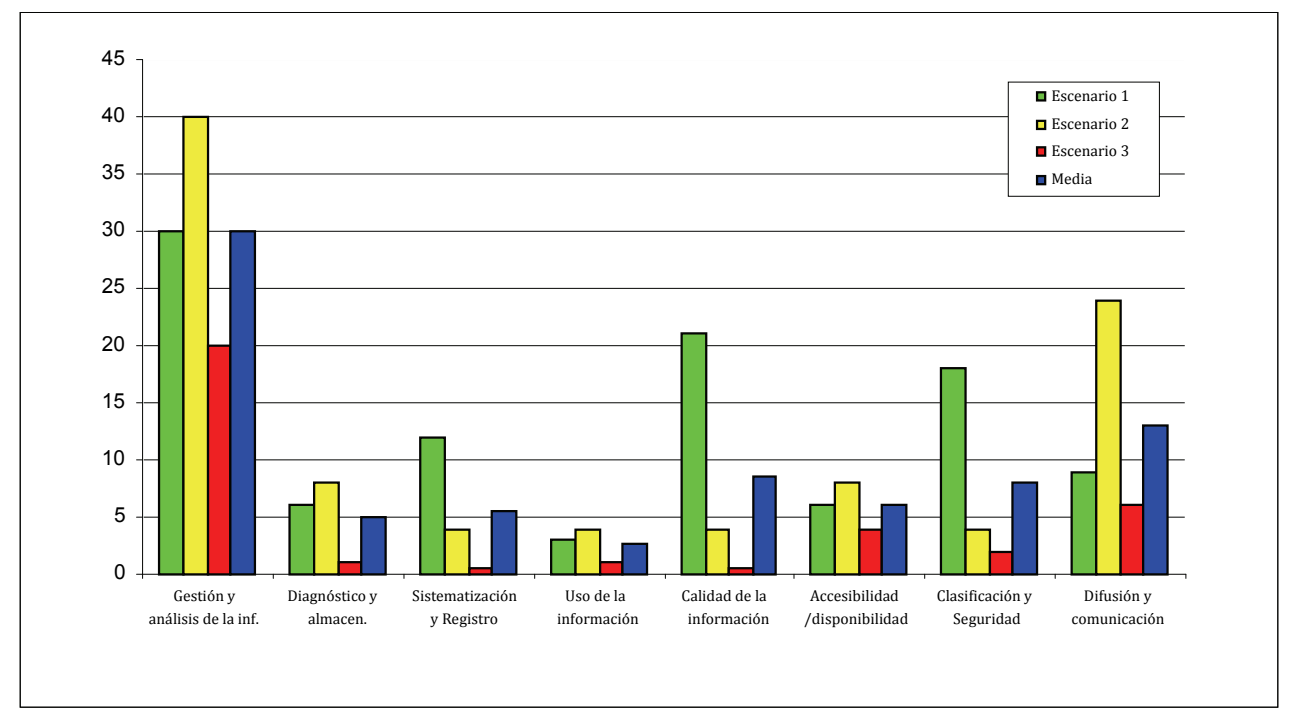

Fig. 4. Necesidades y problemática detectadas en el eje Gestión de Información

Es lógico este resultado ya que el gestionar en primer lugar adecuadamente la información es premisa fundamental para generar y compartir conocimiento.

El siguiente eje identificado fue el de Gestión del conocimiento donde se relacionaron los siguientes temas con las problemáticas y necesidades:

- Aprendizaje Organizacional

- Liderazgo Organizacional

- Cultura Organizacional

- Gerencia del conocimiento

- Impacto del conocimiento

En este eje, se identificó que las personas se preocupan por la forma en que se administra el conocimiento, la cultura organizacional, la forma de liderar los procesos de aprendizaje y el impacto que tiene el conocimiento sobre el bienestar y productividad de las organizaciones. 


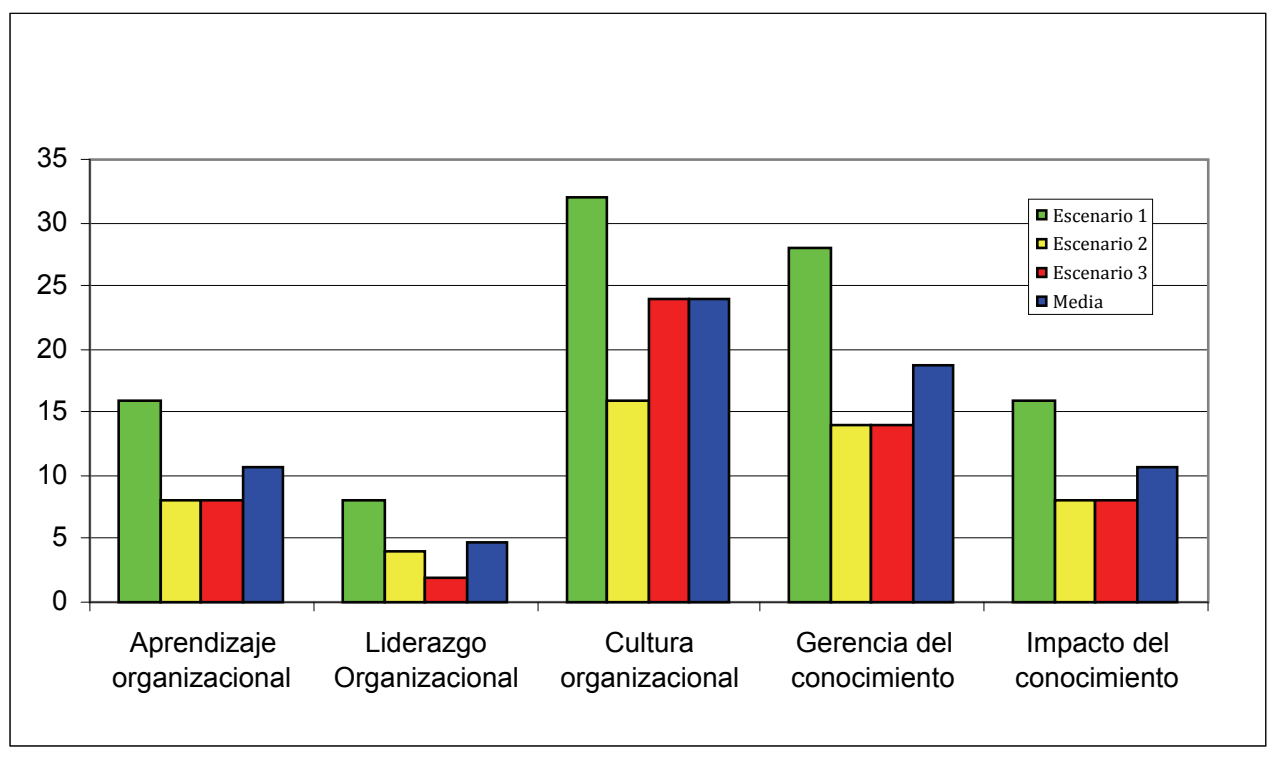

Fig. 5. Necesidades y problemática detectadas en el eje Gestión del Conocimiento

Para el eje de Tecnología quedó demostrado que las personas se interesan por la tecnología en su aplicabilidad y los beneficios que esta pueda representar para el mejoramiento continuo de sus actividades, junto con la ingeniería del conocimiento, la cual se entiende como parte esencial de la tecnología.

Aspectos como las redes de información y el desarrollo de sistemas no llegan a tener la misma relevancia. 


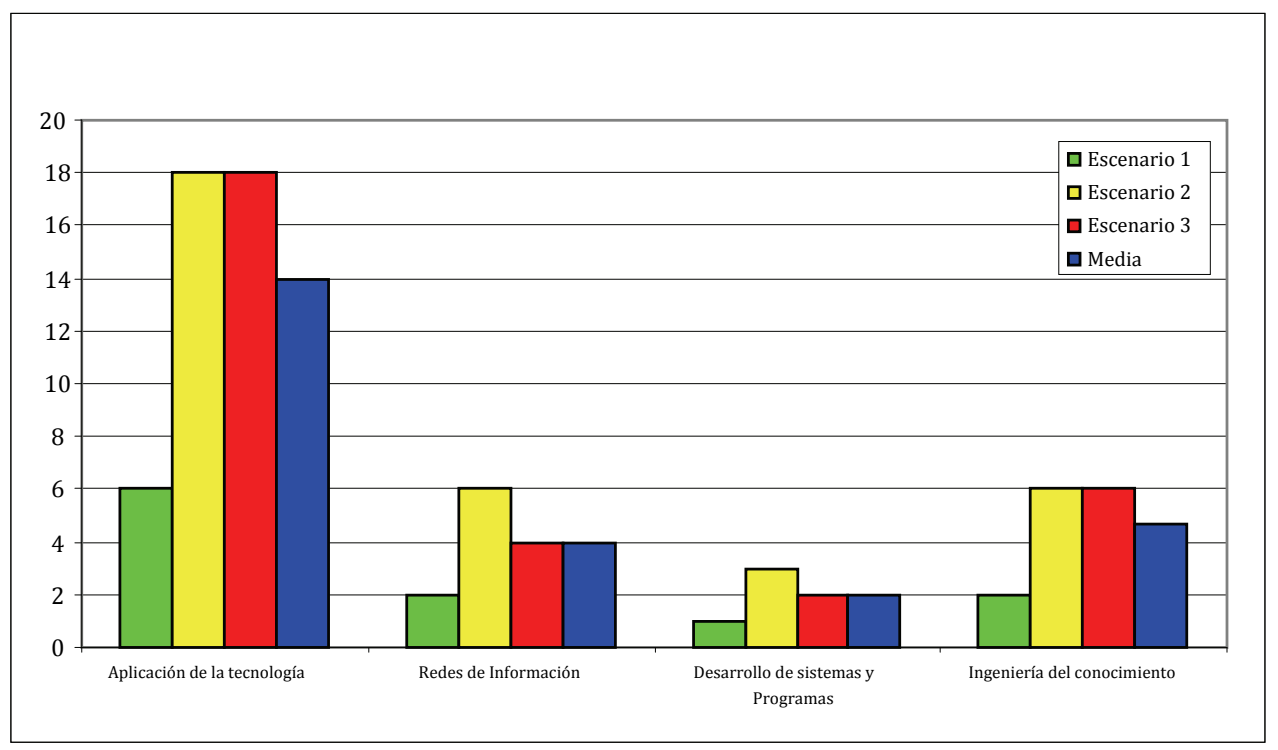

Fig. 6 Necesidades y problemática detectadas en el eje Tecnología

Por último el eje Aprendizaje organizacional, es el que menos socorrido fue por los participantes, debido a que se requiere una madurez tecnológica y organizativa, para que este eje pueda ser implementado a todos los niveles dentro de las organizaciones, cuestiones como la cultura organizaciones, las políticas internas y globales, así como los procedimientos y normas de calidad, son en la mayoría de los casos desconocidas por los miembros de una organización.

Este es el punto principal, sobre el cual las organizaciones deben prestar mayor atención con el fin de tener procesos establecidos, que permitan la capacitación continua y el aprendizaje permanente, que marca la diferencia con otras organizaciones que se han tomado el trabajo de desarrollar estas herramientas corporativas. 


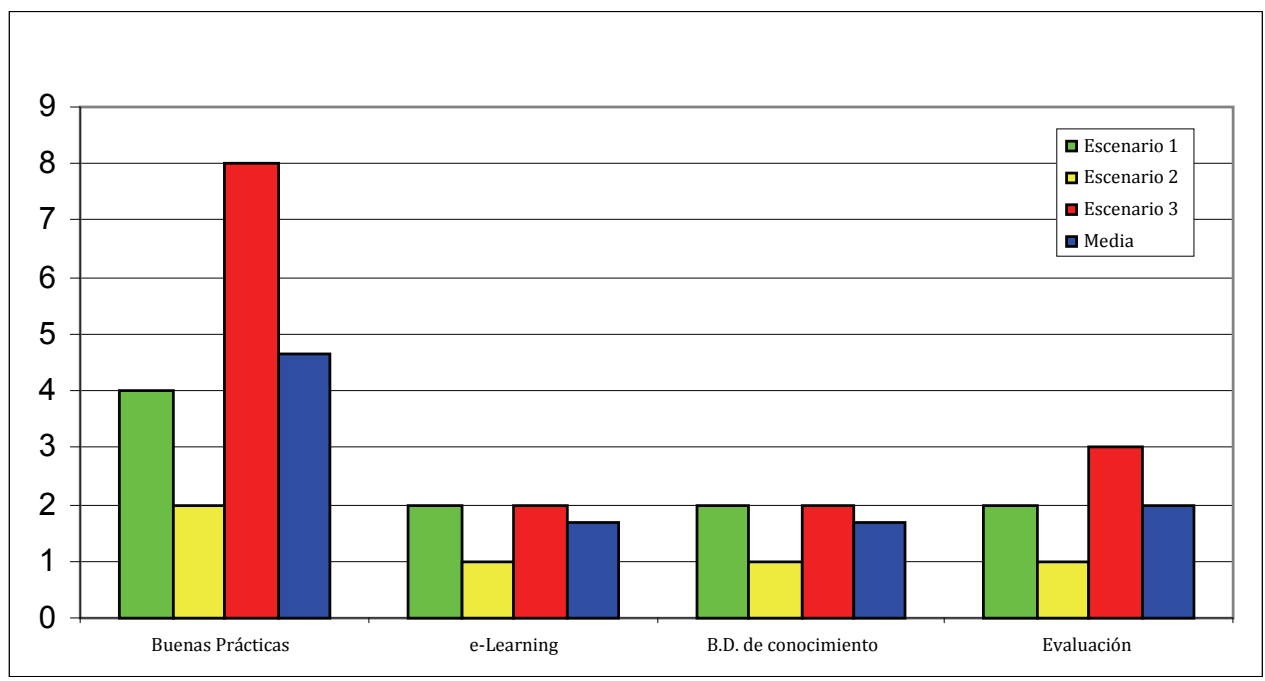

Fig. 7 Necesidades de la problemática detectadas en el eje Aprendizaje

Organizacional

\section{CONCLUSIONES}

De los programas educativos analizados, ninguno se desarrolla a nivel licenciatura, lo que representa un espacio de oportunidad para la carrera que se propone, siendo el nivel maestría es el que mayormente se oferta, seguido por cursos de especialización, diplomados y por último seminarios. No se encontro ninguno a nivel doctorado o nivel post-doctoral

Los programas se desarrollan primordialmente en modalidad presencial, seguido de los programas en línea y por último los semi-presenciales,

Los programas actuales responden a:

- necesidad de preparar directivos de alta calificación para el manejo y aprovechamiento de los recursos tecnológicos e informativos.

- desarrollar vínculos profundos de las empresas con los nuevos paradigmas de las tecnologías de la información y el conocimiento.

- formación profesional de ejecutivos y profesionales que tengan a su cargo el manejo recursos humanos, financieros, materiales o técnicos y que estén preocupados por el manejo de la información dentro de sus organizaciones y por la interacción de los elementos humanos de la misma, siendo estos poseedores de conocimientos relevantes.

Los programas de GC se proponen contribuir a la formación de competencias profesionales de directivos, ejecutivos y profesionales relacionadas con conceptos e instrumentos metodológicos de gestión de la información y el conocimiento y su aplicación en las organizaciones y a mostrar el proceso de transformación que existe 
en las organizaciones actuales, visualizando las tendencias futuras y proporcionando bases para enfrentar los retos, considerando el cambio cultural y tecnológico

Los contenidos de GC están enfocados a:

- Las teorías, procesos y fases de la gestión del conocimiento

- Innovación y competitividad

- Análisis de datos, capital humano

- Comportamiento y aprendizaje organizacional

- Análisis y diseño de sistemas informáticos

- Técnicas de apoyo a la gestión de la información y el conocimiento

- Capital intelectual

- Usabilidad de la información

La problemática y las necesidades detectadas se relacionan fundamentalmente con:

- Procesar de manera eficiente la información que se recibe para uso interno, tanto en tomar esta información como punto de partida para sus labores diarias, como para la toma de decisiones.

- Administrar el conocimiento y la cultura dentro de cada organización de forma que se puedan liderar los procesos de aprendizaje y el impacto que tiene el conocimiento sobre el bienestar y productividad de las personas que forman parte de una organización.

- La tecnología como elemento transformador en la practica de la GC debe ser aplicada de manera adecuada para el mejoramiento continuo de los procesos y actividades en las organizaciones.

- El aprendizaje organizacional es el eje menos desarrollado en las organizaciones ya que requiere madurez tecnológica e inversiones, para que el conocimiento pueda ser compartido y difundido apropiadamente.

\section{REFERENCIAS BIBLIOGRÁFICAS}

AIGNEREN, M. (2002). La Técnica de recolección de información mediante grupos focales. La Sociología en sus escenarios, No. 6 Recuperado el 1 de febrero en: http://aprendeenlinea.udea.edu.co/revistas/index.php/ceo/article/viewArticle/1611 CHAN NÚÑEZ, M.E., (2003) Guía para el diseño curricular por competencias Recuperado el 1 de febrero de 2011 en UDG Virtual: Biblioteca Virtual http://mail.udgvirtual.udg.mx/biblioteca/handle/20050101/1040

CHAN NÚÑEZ, M.E., (2003) El trabajo grupal en el diseño y evaluación curricular Recuperado el 1 de febrero de 2011 en:

http://mail.udgvirtual.udg.mx/biblioteca/bitstream/20050101/1038/1/El_trabajo_grup al_en_el_dise\%C3\%B1o_y_evaluacion_curricular.pdf

DAVENPORT, T. P. (2000). Working knowledge: how organizations manage what they know. Harvard: Harvard Bussines Press.

NONAKA I., Takeuchi. H. (1995). The knowledge-creating company. Oxford: Oxford University Press. 
DUNCKER P. (2007). Innovation a entrepreniurship: practuice and principles . Butterworth-Heinemann.

POLANYI, M. (1998). Personal knowledge: towards a post-critical philosophy. Chicago: University of Chicago Press.

SVEIBY, K. (2000). La nueva riqueza de las empresas. Barcelona: Gestion 2000.

TOBON, S., (2006) Aspectos Básicos de la formación basada en competencias. Talca: Proyecto Massesup Recuperado el 1 de febrero de 2011 en: http://formacioncontinua.sep.gob.mx/sites/cursobasico09/anexos/5Sergio_Tobon.pdf 